\title{
Image Noise Cancellation Using Linear Matrix Inequality and Cellular Neural Network
}

\author{
Cheng-Chih Hou, Cian-Pin Wei, Shih-Chun Huang and Te-Jen Su
}

\begin{abstract}
In this paper, the technique of image noise cancellation is presented by employing cellular neural networks (CNN) and linear matrix inequality (LMI). The main objective is to obtain the templates of $\mathrm{CNN}$ by using a corrupted image and a corresponding desired image. A criterion for the uniqueness and global asymptotic stability of the equilibrium point of $\mathrm{CNN}$ is presented based on the Lyapunov stability theorem (i.e., the feedback template " $\mathrm{A}$ " of $\mathrm{CNN}$ is solved at this step), and the input template " $\mathrm{B}$ " of $\mathrm{CNN}$ is designed to achieve desirable output by using the property of saturation nonlinearity of CNN. It is shown that the problem of image noise cancellation can be characterized in terms of LMIs. The simulation results indicate that the proposed method is useful for practical application.
\end{abstract}

Index Terms-CNN, LMI, global asymptotic stability, Lyapunov.

\section{INTRODUCTION}

Cellular neural networks have been introduced by L.O. Chua and L. Yang [1, 2] in 1988. Due to their local connectivity, cellular neural networks can be applied to image processing and operate at a very high speed in the real time.

The most important key point of investigating CNN is how to find the accurate templates "A" and "B". In recent years, the problems of CNN templates design for image processing have received considerable attention. Genetic algorithm and multilayer CNN were presented to obtain templates for image processing in [3]. In [4], the robustness templates of CNN were designed by using mathematical analysis approach. A CNN with a particular hysteresis nonlinear cell characteristic was employed for image processing in [5, 6]. In practice, a drawback of CNN templates design is that the templates must be simplified to decrease the time of operation [3] or to analyze dynamical behavior in mathematics easily $[4,5,7]$.

Another key point of studying CNN is whether CNN system is stable. Recently, there have been several literatures proposed to deal with the stability of CNN by choosing various Lyapunov functions [8-11].

In general, the criterion of stability for different systems would be represented in form of LMI. In the past few years,

C. C. Hou is with the Department of Electronic Engineering, National Kaohsiung University of Applied Sciences, Kaohsiung, Taiwan 807, R.O.C. (e-mail: 1095320135@cc.kuas.edu.tw)

C. P. Wei is with the Department of Electronic Engineering, National Kaohsiung University of Applied Sciences, Kaohsiung, Taiwan 807, R.O.C.

S. C. Huang is with the Department of Electronic Engineering, National Kaohsiung University of Applied Sciences, Kaohsiung, Taiwan 807, R.O.C.

T. J. Su is with the Department of Electronic Engineering, National Kaohsiung University of Applied Sciences, Kaohsiung, Taiwan 807, R.O.C. (e-mail: sutj@cc.kuas.edu.tw).
LMI has been attracted much attention for their computational tractability and usefulness in control engineering and the number of control problems that can be formulated as LMI problems is large and continue to grow [12]. The LMI can now be solved efficiently by the powerful MATLAB LMI Toolbox [13].

The new strategy of image noise cancellation is proposed by combining CNN and LMI. Without the need for simplifying the templates $[3,4,5,7]$, the proposed method can obtain satisfactory templates to eliminate noise effectively. In this paper, a given smaller size training sample that consists of a corrupted image and a corresponding desired image is presented to find the templates of CNN. A criterion for the uniqueness and global asymptotic stability of the equilibrium point of CNN is derived based on the Lyapunov stability theorem. Next, the input template "B" is obtained by employing the property of saturation nonlinearity of CNN. To combine the above results, it will be shown that the problem of templates design is characterized in form of a standard LMI problem.

The rest of this paper is organized as follows. Section 2 presents formulation and preliminaries for the image noise cancellation problem. In Section 3, the problem of stability for CNN system is discussed and the templates design of CNN for the image noise cancellation is characterized by LMIs. An example is given in Section 4 to demonstrate the proposed methodology. Finally, conclusion is drawn in Section 5.

\section{Problem Formulation AND PRELIMINARIES}

In this paper, the space-invariant CNN system and LMI are utilized for image noise cancellation. The block diagram for the training system is shown in Fig. 1. The templates of CNN are trained by a given training sample with smaller size from LMI perspective and then the CNN with given templates is employed to eliminate noise from arbitrary larger corrupted images arbitrary larger corrupted images.

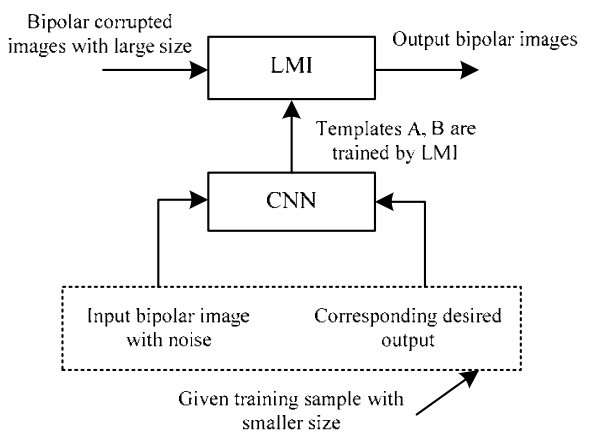

Figure 1. The training system 
The system structure of a cell of the space-invariant CNN is depicted in Fig. 2, where $\mathrm{U}$ is the input image with size $\mathrm{M}$ by $\mathrm{N}, \mathrm{Y}$ is the output image with size is equal to input image, $x_{i j}$ is the state of cell $(i, j), u_{i j}$ is the pixel of input image at $i_{\text {th }}$ row and $j_{\text {th }}$ column, $y_{i j}$ is the pixel of output image at $i_{\text {th }}$ row and $j_{\text {th }}$ column, $\mathrm{z}$ is the threshold, $\mathrm{A}$ is the feedback template, and $\mathrm{B}$ is the input template.

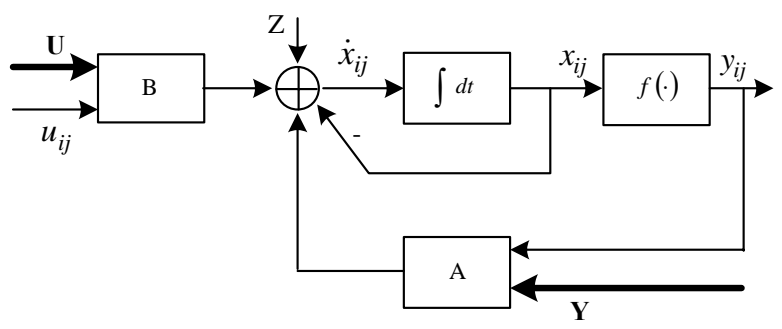

Figure 2. The system structure of cell $(i, j)$

The dynamical behavior of cell $(i, j)$ is represented as follows [7]

$$
\begin{aligned}
& \dot{x}_{i j}(t)=-x_{i j}(t)+A \otimes Y_{i j}(t)+B \otimes U_{i j}(t)+z(t) \\
& y_{i j}\left(x_{i j}(t)\right)=f\left(x_{i j}(t)\right)
\end{aligned}
$$

where the symbol “ $\otimes$ ” denotes the summation of dot products, and

$$
y_{i j}\left(x_{i j}(t)\right)=f\left(x_{i j}(t)\right)=0.5\left(\left|x_{i j}(t)+1\right|-\left|x_{i j}(t)-1\right|\right) \quad \forall i, j
$$

After repacking, the dynamical behavior of a space-invariant continuous time CNN can be described by the following equation

$$
\begin{aligned}
& \dot{\mathbf{x}}(t)=-\mathbf{x}(t)+\hat{\mathbf{A}} \mathbf{y}(\mathbf{x}(t))+\hat{\mathbf{B}} \mathbf{u}(t)+\mathbf{z}(t) \\
& \mathbf{y}(\mathbf{x}(t))=\mathbf{f}(\mathbf{x}(t))
\end{aligned}
$$

or

$$
\Rightarrow \frac{d x_{i}(t)}{d t}=-x_{i}(t)+\sum_{j=1}^{n} \hat{a}_{i j} y_{j}\left(x_{j}(t)\right)+\sum_{j=1}^{n} \hat{b}_{i j} u_{j}(t)+z(t)
$$$$
y_{i}\left(x_{i}(t)\right)=f\left(x_{i}(t)\right)
$$

, where $\mathbf{x}=\left[\begin{array}{llll}x_{1} & x_{2} & \cdots & x_{n}\end{array}\right]^{T}$ is the sate vector $(\mathrm{n}=\mathrm{MN})$, $\hat{\mathbf{A}}=\left\{\hat{a}_{i j}\right\}$ is the feedback matrix, $\hat{\mathbf{B}}=\left\{\hat{b}_{i j}\right\}$ is the input matrix, $\mathbf{y}(\mathbf{x}(\cdot))=\left[\begin{array}{llll}y_{1}\left(x_{1}(\cdot)\right) & y_{2}\left(x_{2}(\cdot)\right) & \cdots & y_{n}\left(x_{n}(\cdot)\right)\end{array}\right]^{T} \quad$ is the output vector, $\mathbf{u}=\left[\begin{array}{llll}u_{1} & u_{2} & \cdots & u_{n}\end{array}\right]^{T}$ is the input vector, $\mathbf{z}=\left[\begin{array}{llll}z & Z & \cdots & z\end{array}\right]^{T}$ that would be ignored in this method is the threshold vector, and

$$
y_{i}\left(x_{i}(t)\right)=f\left(x_{i}(t)\right)=0.5\left(\left|x_{i}(t)+1\right|-\left|x_{i}(t)-1\right|\right) \quad \forall i
$$

Remark: The templates " $A$ " and " $B$ " are different from the feedback matrix $\hat{\mathbf{A}}$ and input matrix $\hat{\mathbf{B}}$ respectively, but their relation is quite close [7].

In this paper, the templates are trained by a corrupted image (be represented as the static input vector $\mathbf{u}$ ) and a corresponding desired image (be represented as the output vector $\mathbf{y}^{*}$ that we want at steady state). According to above description, it is obvious that (3) can be rewritten as

$\dot{\mathbf{x}}(t)=-\mathbf{x}(t)+\hat{\mathbf{A}} \mathbf{y}(\mathbf{x}(t))+\hat{\mathbf{u}}$

$\mathbf{y}(\mathbf{x}(t))=\mathbf{f}(\mathbf{x}(t))$ or

$$
\begin{aligned}
& \Rightarrow \frac{d x_{i}(t)}{d t}=-x_{i}(t)+\sum_{j=1}^{n} \hat{a}_{i j} y_{j}\left(x_{j}(t)\right)+\hat{u}_{i} \\
& y_{i}\left(x_{i}(t)\right)=f\left(x_{i}(t)\right)
\end{aligned}
$$

where $\hat{\mathbf{u}}=\left[\begin{array}{llll}\hat{u}_{1} & \hat{u}_{2} & \cdots & \hat{u}_{n}\end{array}\right]^{T}=\hat{\mathbf{B}} \mathbf{u}$ is independent on time.

In order to simplify the proof of the stability of CNN, we will shift the equilibrium point $\mathbf{x}^{*}=\left[\begin{array}{llll}x_{1}^{*} & x_{2}^{*} & \cdots & x_{n}^{*}\end{array}\right]^{T}$ of (5) to the origin. Let $s_{i}(t)=x_{i}(t)-x_{i}^{*}$, system (5) can be represented as

$$
\begin{aligned}
& \dot{\mathbf{s}}(t)=-\mathbf{s}(t)+\hat{\mathbf{A}} \mathbf{\Phi}(\mathbf{s}(t)) \\
& \mathbf{y}\left(\mathbf{s}(t)+\mathbf{x}^{*}\right)=\mathbf{f}\left(\mathbf{s}(t)+\mathbf{x}^{*}\right)
\end{aligned}
$$

or

$\Rightarrow \frac{d s_{i}(t)}{d t}=-s_{i}(t)+\sum_{j=1}^{n} \hat{a}_{i j} \phi_{j}\left(s_{j}(t)\right)$

$y_{i}\left(s_{i}(t)+x_{i}^{*}\right)=f\left(s_{i}(t)+x_{i}^{*}\right)$

where $\mathbf{s}=\left[\begin{array}{llll}s_{1} & s_{2} & \cdots & s_{n}\end{array}\right]^{T}$ is the state vector,

$\boldsymbol{\Phi}(\mathbf{s}(t))=\left[\begin{array}{llll}\phi_{1}\left(s_{1}(t)\right) & \phi_{2}\left(s_{2}(t)\right) & \cdots & \phi_{n}\left(s_{n}(t)\right)\end{array}\right]^{T}$

$\phi_{i}\left(s_{i}(t)\right)=y_{i}\left(s_{i}(t)+x_{i}^{*}\right)-y_{i}\left(x_{i}^{*}\right),\left|\phi_{i}\left(s_{i}(t)\right)\right| \leq\left|s_{i}(t)\right|$, and

$\phi_{i}^{2}\left(s_{i}(t)\right) \leq s_{i}(t) \phi_{i}\left(s_{i}(t)\right), \forall i$

or

$$
\boldsymbol{\Phi}^{T}(\mathbf{s}(t)) \boldsymbol{\Phi}(\mathbf{s}(t)) \leq \mathbf{s}^{T}(t) \boldsymbol{\Phi}(\mathbf{s}(t))
$$

The following lemma that will be frequently used in the derivation of our main result is so-called Schur Complement Lemma.

Lemma 1 [14]: Given constant matrices M, L, and R of appropriate dimensions where $\mathrm{M}$ and $\mathrm{R}$ are symmetric and $\mathrm{R}>0$, then $M+L^{T} R L<0$ if and only if

$$
\left[\begin{array}{cc}
M & L^{T} \\
L & -R^{-1}
\end{array}\right]<0 \text { or }\left[\begin{array}{cc}
-R^{-1} & L \\
L^{T} & M
\end{array}\right]<0 \text {. }
$$

\section{MAin Result AND PROOF}

According to the training system (see Fig. 1), we will first derive a criterion for the uniqueness and global asymptotic stability of the equilibrium point of the CNN based on the Lyapunov stability theorem (i.e., "A" template of CNN is solved). Afterward using the mathematical analysis approach, "B" template of CNN is designed to achieve desirable output $\mathbf{y}^{*}$ at steady state.

Theorem 1: Consider the training system in Fig. 1 and the dynamical behavior of CNN (3), if the training sample that consists of a bipolar corrupted image and its corresponding desired bipolar image is given and the following LMIs are existence, then the templates of the $\mathrm{CNN}$ for the image reconstruction would be solvable

(i) $-\hat{\mathbf{A}}-\hat{\mathbf{A}}^{T}>0$

(ii) $\sum_{j=1}^{n} \hat{a}_{i j} y_{j}^{*}+\sum_{j=1}^{n} \hat{b}_{i j} u_{j}-1>0$ if $y_{i}^{*}=1\left(y_{i}^{*}\right.$ is white) 
Proceedings of the International MultiConference of Engineers and Computer Scientists 2008 Vol I IMECS 2008, 19-21 March, 2008, Hong Kong

$$
\begin{aligned}
& -\left(\sum_{j=1}^{n} \hat{a}_{i j} y_{j}^{*}+\sum_{j=1}^{n} \hat{b}_{i j} u_{j}+1\right)>0 \text { if } y_{i}^{*}=-1\left(y_{i}^{*} \text { is black }\right) \\
& \mathrm{i}=1,2,3, \ldots, \mathrm{MN}
\end{aligned}
$$

where $\hat{\mathbf{A}}=\left\{\hat{a}_{i j}\right\}, \hat{\mathbf{B}}=\left\{\hat{b}_{i j}\right\}, u_{i}$ that depends on the corrupted image of training sample and $y_{i}^{*}$ that depends on the corresponding desired image of training sample is the given binary output vector at steady state.

Note: The image is coded such that -1 corresponds to black pixels and +1 to white ones.

Proof:

First, herein the proof of the criterion for the uniqueness and global asymptotic stability of the equilibrium point of the $\mathrm{CNN}$ shall be accomplished. In order to prove the global asymptotic stability of the origin of (6), we choose the following positive definite Lyapunov functional according to [8-11]:

$$
V(\mathbf{s}(t))=\mathbf{s}^{T}(t) \mathbf{s}(t)+2 \alpha \sum_{i=1}^{n} \int_{0}^{s_{i}(t)} \phi_{i}(\ell) d \ell
$$

where $\alpha$ is a positive constant.

The time derivative of $V(\mathbf{s}(t))$ along the trajectories of (6) is obtained as

$$
\begin{aligned}
\dot{V}(\mathbf{s}(t))= & -2 \mathbf{s}^{T}(t) \mathbf{s}(t)+2 \boldsymbol{\Phi}^{T}(\mathbf{s}(t)) \hat{\mathbf{A}}^{T} \mathbf{s}(t)-2 \alpha \mathbf{\Phi}^{T}(\mathbf{s}(t)) \mathbf{s}(t) \\
& +2 \alpha \mathbf{\Phi}^{T}(\mathbf{s}(t)) \hat{\mathbf{A}} \boldsymbol{\Phi}(\mathbf{s}(t)) \\
= & -2 \mathbf{s}^{T}(t) \mathbf{s}(t)+2 \boldsymbol{\Phi}^{T}(\mathbf{s}(t)) \hat{\mathbf{A}}^{T} \mathbf{s}(t)+2 \alpha \mathbf{\Phi}^{T}(\mathbf{s}(t)) \hat{\mathbf{A}} \boldsymbol{\Phi}(\mathbf{s}(t)) \\
& -2 \alpha \mathbf{\Phi}^{T}(\mathbf{s}(t)) \boldsymbol{\Phi}(\mathbf{s}(t))-2 \alpha \mathbf{\Phi}^{T}(\mathbf{s}(t))[\mathbf{s}(t)-\boldsymbol{\Phi}(\mathbf{s}(t))]
\end{aligned}
$$

According to (7), we can write

$$
\begin{aligned}
\dot{V}(\mathbf{s}(t)) \leq & -2 \mathbf{s}^{T}(t) \mathbf{s}(t)+2 \boldsymbol{\Phi}^{T}(\mathbf{s}(t)) \hat{\mathbf{A}}^{T} \mathbf{s}(t)+2 \alpha \mathbf{\Phi}^{T}(\mathbf{s}(t)) \hat{\mathbf{A}} \boldsymbol{\Phi}(\mathbf{s}(t)) \\
= & -2 \mathbf{s}^{T}(t) \mathbf{s}(t)+2 \boldsymbol{\Phi}^{T}(\mathbf{s}(t)) \hat{\mathbf{A}}^{T} \mathbf{s}(t)+\alpha \boldsymbol{\Phi}^{T}(\mathbf{s}(t)) \hat{\mathbf{A}} \boldsymbol{\Phi}(\mathbf{s}(t)) \\
& +\alpha \boldsymbol{\Phi}^{T}(\mathbf{s}(t)) \hat{\mathbf{A}}^{T} \boldsymbol{\Phi}(\mathbf{s}(t))
\end{aligned}
$$

The inequality (13) can be rewritten as

$$
\dot{V}(\mathbf{s}(t)) \leq\left[\begin{array}{ll}
\mathbf{s}^{T}(t) & \mathbf{\Phi}^{T}(\mathbf{s}(t))
\end{array}\right]\left[\begin{array}{cc}
-2 I & \hat{\mathbf{A}} \\
\hat{\mathbf{A}}^{T} & \alpha\left(\hat{\mathbf{A}}+\hat{\mathbf{A}}^{T}\right)
\end{array}\right]\left[\begin{array}{c}
\mathbf{s}(t) \\
\mathbf{\Phi}(\mathbf{s}(t))
\end{array}\right]
$$

From (14), if

$\left[\begin{array}{cc}-2 I & \hat{\mathbf{A}} \\ \hat{\mathbf{A}}^{T} & \alpha\left(\hat{\mathbf{A}}+\hat{\mathbf{A}}^{T}\right)\end{array}\right]<0$

, then the time derivative of $V(\mathbf{s}(t))$ is also negative definite.

Using the Lemma 1 (Schur Complement Lemma), the inequality (15) holds if and only if

$\alpha\left(\hat{\mathbf{A}}+\hat{\mathbf{A}}^{T}\right)+\frac{1}{2} \hat{\mathbf{A}}^{T} \hat{\mathbf{A}}<0$

or $\alpha\left(-\hat{\mathbf{A}}-\hat{\mathbf{A}}^{T}\right)-\frac{1}{2} \hat{\mathbf{A}}^{T} \hat{\mathbf{A}}>0$

By choosing $\alpha>\frac{\lambda_{1}}{\lambda_{2}}$, where $\lambda_{1}$ is the maximum eigenvalue of $\frac{1}{2} \hat{\mathbf{A}}^{T} \hat{\mathbf{A}}$ and $\lambda_{2}$ is the minimum eigenvalue of $-\hat{\mathbf{A}}-\hat{\mathbf{A}}^{T}$, (16) can be simplified as

$$
\hat{\mathbf{A}}+\hat{\mathbf{A}}^{T}<0 \text { or }-\hat{\mathbf{A}}-\hat{\mathbf{A}}^{T}>0
$$

In the above, we have demonstrated that (17) is the criterion of the global asymptotic stability of the origin of (6). Now, the uniqueness of the equilibrium point $\mathbf{s}^{*}=\mathbf{0}$ is proved by contradiction method. Consider the equilibrium equation of (6)

$\mathbf{0}=-\mathbf{s}^{*}+\hat{\mathbf{A}} \boldsymbol{\Phi}\left(\mathbf{s}^{*}\right)$

where $\mathbf{s}^{*}$ is the equilibrium point. From (18), it is clearly that if $\boldsymbol{\Phi}\left(\mathbf{s}^{*}\right)=\mathbf{0}$, then $\mathbf{s}^{*}=\mathbf{0}$. Let $\boldsymbol{\Phi}\left(\mathbf{s}^{*}\right) \neq \mathbf{0}$, multiplying both sides of (18) by $2 \boldsymbol{\Phi}^{T}\left(\mathbf{s}^{*}\right)$, we obtain

$$
\mathbf{0}=-2 \boldsymbol{\Phi}^{T}\left(\mathbf{s}^{*}\right) \mathbf{s}^{*}+2 \boldsymbol{\Phi}^{T}\left(\mathbf{s}^{*}\right) \hat{\mathbf{A}} \boldsymbol{\Phi}\left(\mathbf{s}^{*}\right)
$$

The equation (19) can be rewritten as

$$
\begin{aligned}
2 \boldsymbol{\Phi}^{T}\left(\mathbf{s}^{*}\right) \hat{\mathbf{A}} \boldsymbol{\Phi}\left(\mathbf{s}^{*}\right) & =2 \boldsymbol{\Phi}^{T}\left(\mathbf{s}^{*}\right) \mathbf{s}^{*} \\
& =2 \boldsymbol{\Phi}^{T}\left(\mathbf{s}^{*}\right)\left[\mathbf{s}^{*}-\boldsymbol{\Phi}\left(\mathbf{s}^{*}\right)\right]+2 \boldsymbol{\Phi}^{T}\left(\mathbf{s}^{*}\right) \boldsymbol{\Phi}\left(\mathbf{s}^{*}\right)
\end{aligned}
$$

According to (7), equation (20) can be expressed as the following inequality

$$
\boldsymbol{\Phi}^{T}\left(\mathbf{s}^{*}\right)\left(\hat{\mathbf{A}}+\hat{\mathbf{A}}^{T}\right) \boldsymbol{\Phi}\left(\mathbf{s}^{*}\right) \geq 0
$$

Consider the criterion for the global asymptotic stability of CNN (17), it implies that

$$
\boldsymbol{\Phi}^{T}\left(\mathbf{s}^{*}\right)\left(\hat{\mathbf{A}}+\hat{\mathbf{A}}^{T}\right) \boldsymbol{\Phi}\left(\mathbf{s}^{*}\right)<0
$$

because equation (21) contradicts with equation (22), the equilibrium point $\mathbf{s}^{*}=0$ of (6) is unique. Thus, (5) has a unique equilibrium point for every $\hat{\mathbf{u}}$.

So far, the criterion of uniqueness and global asymptotic stability of the equilibrium point of CNN has been derived above. In other words, the template " $\mathrm{A}$ " is obtained already according to (9). Now, we will design template "B" of CNN to achieve desirable output $\mathbf{y}^{*}$ at steady state. From the training system in Fig. 1 and the dynamical behavior of CNN in (5), the equilibrium equation of (5) is shown as

$$
\begin{aligned}
& \mathbf{0}=-\mathbf{x}^{*}+\hat{\mathbf{A}} \mathbf{y}\left(\mathbf{x}^{*}\right)+\hat{\mathbf{B}} \mathbf{u} \\
& \mathbf{y}^{*}=\mathbf{y}\left(\mathbf{x}^{*}\right)=\mathbf{f}\left(\mathbf{x}^{*}\right) \\
& \text { or }
\end{aligned}
$$

$$
\begin{aligned}
& \Rightarrow 0=-x_{i}^{*}+\sum_{j=1}^{n} \hat{a}_{i j} y_{j}\left(x_{j}^{*}\right)+\sum_{j=1}^{n} \hat{b}_{i j} u_{j} \\
& y_{i}^{*}=y_{i}\left(x_{i}^{*}\right)=f\left(x_{i}^{*}\right)
\end{aligned}
$$

Where

$\mathbf{u}$ : The input vector that depends on the bipolar corrupted image of training sample.

$\mathbf{y}^{*}$ : The output vector at steady state that depends on the corresponding desired image of training sample.

$\hat{\mathbf{A}}$ : The feedback matrix that can be obtained by (9).

$\hat{\mathbf{B}}$ : The input matrix that should be calculated at this step.

By using the property of saturation nonlinearity (4), (23) is rewritten as the following inequalities

$$
\begin{gathered}
\sum_{j=1}^{n} \hat{a}_{i j} y_{j}^{*}+\sum_{j=1}^{n} \hat{b}_{i j} u_{j} \geq 1 \text { if } y_{i}^{*}=1 \\
\sum_{j=1}^{n} \hat{a}_{i j} y_{j}^{*}+\sum_{j=1}^{n} \hat{b}_{i j} u_{j} \leq-1 \text { if } y_{i}^{*}=-1 \\
\mathrm{i}=1,2,3, \ldots, \mathrm{MN}
\end{gathered}
$$

Rearranging (24), we obtain 


$$
\begin{aligned}
& \sum_{j=1}^{n} \hat{a}_{i j} y_{j}^{*}+\sum_{j=1}^{n} \hat{b}_{i j} u_{j}-1>0 \text { if } y_{i}^{*}=1 \\
& -\left(\sum_{j=1}^{n} \hat{a}_{i j} y_{j}^{*}+\sum_{j=1}^{n} \hat{b}_{i j} u_{j}+1\right)>0 \text { if } y_{i}^{*}=-1 \\
& \quad i=1,2,3, \ldots, \mathrm{MN} \quad \text { i.e., the inequality (10) holds. }
\end{aligned}
$$

Combination of (17) and (25) forms the main result in this chapter, and it will be employed to eliminate noise from other corrupted images.

\section{EXAMPLE}

In this section, an example is presented to illustrate the effectiveness of the proposed methodology. Herein we will use the different bipolar training samples (with smaller size 16 by 16) to train the templates of CNN based on theorem 1 . Then the CNN with given templates would be employed to eliminate noise from other corrupted images (with larger size 128 by 128$)$.

Firstly, the bipolar training sample (see Fig. 3) that consists of a corrupted image with $5 \%$ noise and its corresponding desired image is utilized to train the templates of CNN.

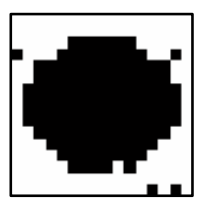

(a)

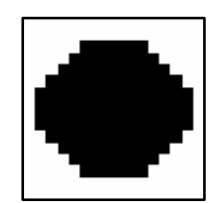

(b)
Figure 3. Training sample

(a) corrupted image with 5\% noise (b) desired image

\begin{tabular}{|c|c|c|c|}
\hline \multirow{3}{*}{$A_{5 \%}=$} & -10.0718 & 7.2009 & 10.8975 \\
\hline & -0.4159 & -9.3977 & 0.8350 \\
\hline & -10.3008 & -7.2822 & 10.3225 \\
\hline \multirow{3}{*}{$\mathrm{B}_{5 \%}=$} & 22.7446 & 3.5208 & 0.6006 \\
\hline & 11.8485 & 24.1257 & 10.5424 \\
\hline & 21.6440 & 5.9648 & 1.8121 \\
\hline
\end{tabular}

According to theorem 1, we obtain the templates $A_{5 \%}$ and $\mathrm{B}_{5 \%}$ as follows

Now, we consider another corrupted image with $10 \%$ noise and its corresponding desired image shown in Fig. 4.

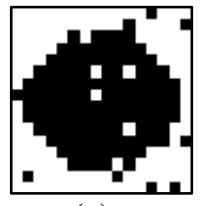

(a)

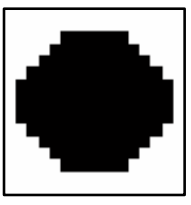

(b)
Figure 4. Training sample

(a) corrupted image with $10 \%$ noise (b) desired image

The corresponding templates of $\mathrm{CNN}$ are obtained by using theorem 1 .

$\mathrm{A}_{10 \%}=$\begin{tabular}{|c|c|c|}
\hline-2.8107 & 6.0507 & -8.9027 \\
\hline-2.0066 & -9.4413 & 3.0729 \\
\hline 9.1743 & -5.8931 & 3.2050 \\
\hline
\end{tabular}

$\mathrm{B}_{10 \%}=$\begin{tabular}{|c|c|c|}
\hline 8.1918 & 19.6384 & 18.5049 \\
\hline 10.4013 & 17.6569 & 9.1306 \\
\hline 13.5312 & 23.8828 & 5.1621 \\
\hline
\end{tabular}

After the calculation of the templates, the LENA images with various noise ratios are applied to the input of CNN and we will obtain the reconstruction images at the output of CNN. When the noise ration of input image is $5 \%$, the images before and after CNN processing are shown in Fig. 5. Fig. 6 shows the results when the noise ration of input image is $10 \%$.

In order to calculate the performance of the presented method under different levels of noise ratio, we introduce the reconstruction error rate as

$\gamma=\frac{\sum_{i=1}^{M} \sum_{j=1}^{N}\left|\tilde{y}_{i j}-\hat{y}_{i j}\right|}{2 M N} \times 100 \%$

where $\tilde{y}_{i j}$ is the pixel of the ideal image as shown in Fig. 5 (a) or in Fig. 6 (a), $\hat{y}_{i j}$ is the pixel of the reconstruction image at the output of CNN, and $\mathrm{M}$ and $\mathrm{N}$ are the horizontal and vertical horizons.

Further, the reconstruction error rate will be compared with the noise ratio of the input corrupted image by the following index

$\eta=\frac{\text { noise ratio of the corrupted image }-\gamma}{\text { noise ratio of the corrupted image }} \times 100 \%$

It is obvious that the smaller $\gamma$ and larger $\eta$ are better, and we will compare the performance of CNN with various templates by these values. The $\gamma$ and the $\eta$ values for various templates under different levels of noise ratio are shown in Fig. 7 and Fig. 8 respectively.

From the Fig. 5 and Fig. 6, they show that the reconstruction output images of the proposed CNN are very satisfactory. Further, the results in Fig. 5 and Fig. 6 also show that the templates trained by the training sample with higher noise ratio outperform the templates trained by lower noise ratio. In Fig. 7 and Fig. 8, they reveal that the proposed CNN can eliminate noise effectively and about the ninety percent of the noise of the corrupted images have been removed regardless of the noise ratio.

\section{CONCLUSION}

In this paper, a solution to the templates design of CNN for noise cancellation is proposed. It is shown that the design problem can be transformed into LMIs, and then it is straightforward to obtain the solution by the recently developed LMI Toolbox. Hence, we have presented an effective design method and computational procedures to the templates design for image reconstruction. In contrast to traditional methods (i.e., simplifying the design templates), the proposed one can obtain satisfactory templates to eliminate noise effectively, without the need for simplifying templates.

From the simulated results, we find that the reconstruction images and the reconstruction error rate are very satisfactory. Thus, the new strategy provides a worthy approach for the 
Proceedings of the International MultiConference of Engineers and Computer Scientists 2008 Vol I IMECS 2008, 19-21 March, 2008, Hong Kong

noise cancellation problem. In the future, the problem of robust templates design should be considered under the CNN system with uncertain.

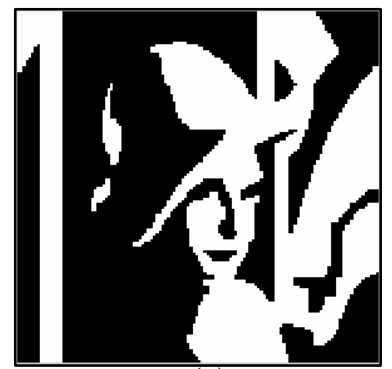

(a)

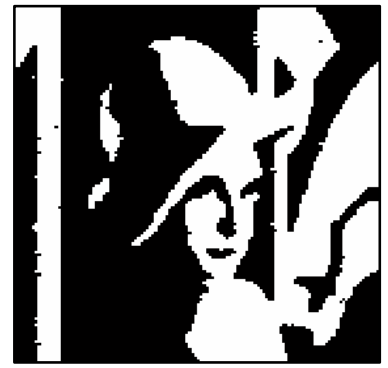

(c)

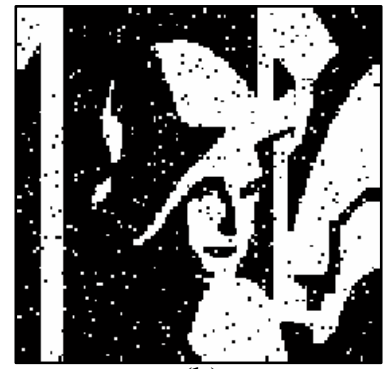

(b)

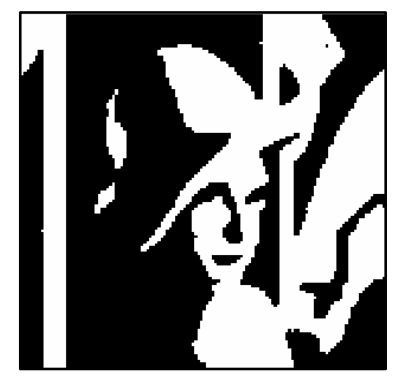

(d)
Figure 5. Noise ratio in 5\%

(a) Ideal image (b) Image before CNN processing (c) Image after $C N N$ processing with templates $A_{5 \%}$ and $B_{5 \%}$ (d) Image after CNN processing with templates $\mathrm{A}_{10 \%}$ and $\mathrm{B}_{10 \%}$

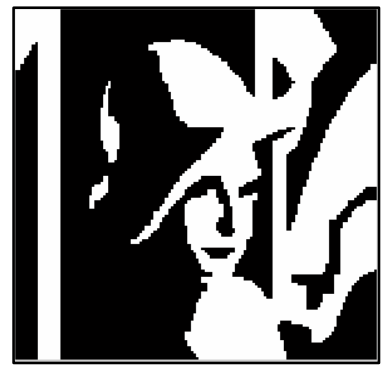

(a)

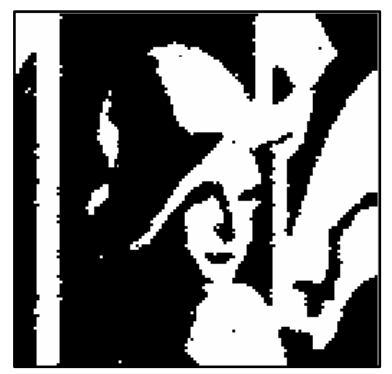

(c)

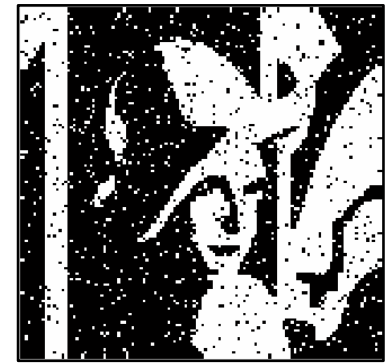

(b)

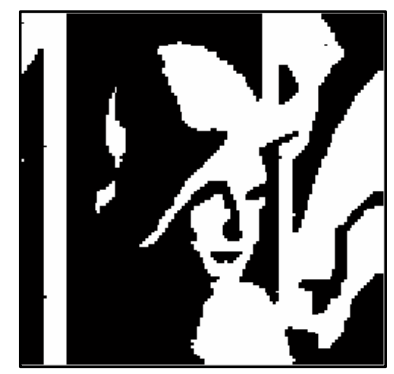

(d)
Figure 6. Noise ratio in $10 \%$

(a) Ideal image (b) Image before CNN processing (c) Image after $C N N$ processing with templates $A_{5 \%}$ and $B_{5 \%}$ (d) Image after CNN processing with templates $\mathrm{A}_{10 \%}$ and $\mathrm{B}_{10 \%}$

\section{REFERENCES}

[1] Chua, L. O. and Yang, L.: "Cellular neural networks: theory,” IEEE Trans. Circuits and Systems, Oct. 1988, 35, pp. 1257-1272

[2] Chua, L. O. and Yang, L.: "Cellular neural networks: applications," IEEE Trans. on Circuits and Systems, Oct. 1988, 35, pp.1273-1290

[3] Lopez, P. D., Vilarino, L.: and Cabello, D.: "Design of multilayer discrete time cellular neural networks for image processing tasks based on genetic algorithms," IEEE International Symposium on Circuits

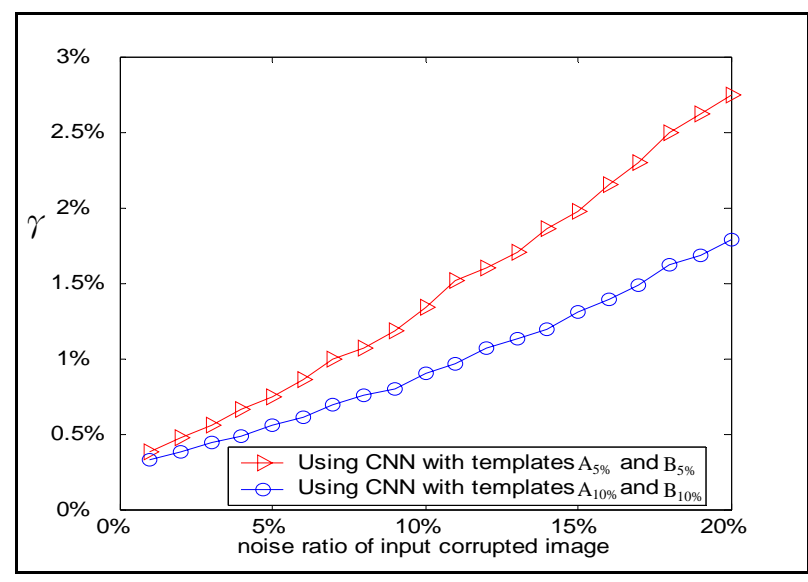

Figure 7. Comparison of $\gamma$ values among the various templates of CNN

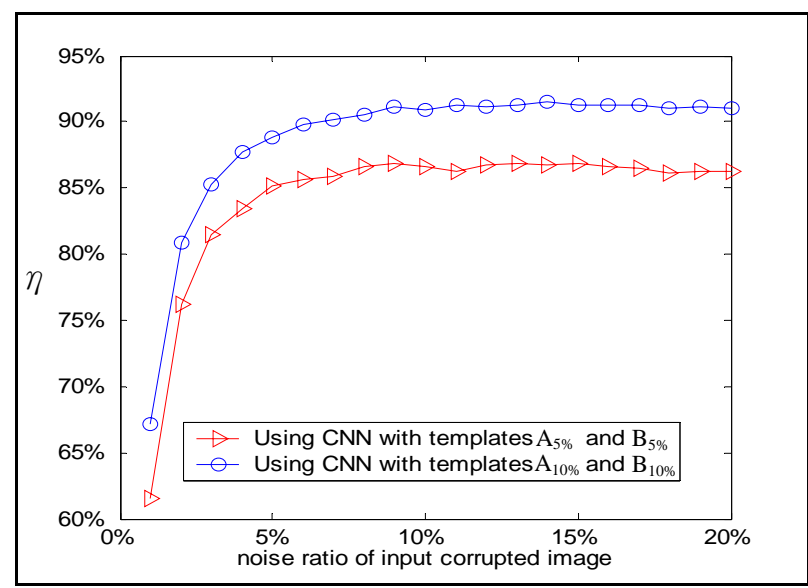

Figure 8. Comparison of $\eta$ values among the various templates

and Systems, May 2000, 4, pp. 133-136

[4] Ming, L. and Min, L.: "The robustness design of templates of CNN for detecting inner corners of objects in gray-scale images," IEEE International Conference on Communications, Circuits and Systems, June 2004, 2, pp. 1090-1093

[5] Matei, R. P.: "Image processing using hysteretic cellular neural networks," IEEE International Symposium on Circuits and Systems, May 2000, 4, pp. 129-132

[6] Gupta, N. K. and Hidemitsu, O.: "Optoelectronic architecture for cellular neural networks based on a new bias method and spatial coding scheme," Optics Communications, April 1997, 138, pp.11-15

[7] Chua, L. O. and Roska, T.: "Cellular neural networks and visual computing," (New York: Cambridge University Press, 2002)

[8] Cao, J.: "Global stability conditions for delayed CNNs," IEEE Trans. on Circuits and Systems-I: Fundamental Theory and Applications, Nov. 2001, 48, pp. 1330-1333

[9] Singh, V.: "Global asymptotic stability of cellular neural networks with unequal delays: LMI approach,” Electronics Letters, April 2004, 40, pp. 548-549

[10] Arik, S. and Tavsanoglu, V.: "On the global asymptotic stability of delayed cellular neural networks," IEEE Trans. on Circuits and Systems-I: Fundamental Theory and Applications, April 2000, 47, pp. 571-574

[11] Gilli, M.: "A Lyapunov function approach to the study of the stability of cellular neural networks," IEEE International Symposium on Circuits and Systems, May 1993, 4, pp. 2584-2587

[12] Boyd, S., Ghaoui, L. E., Feron, E. and Balakrishnan, V.: "Linear matrix inequalities in system and control theory,” (Philadelphia: Society for Industrial and Applied Mathematics, 1994)

[13] Gahinet, P. Nemirovskii, A. Laub, A. and Chilali, M.: "LMI Control Toolbox: For Use With MATLAB,” (The MATH Works Inc., 1995)

[14] Wang, Z. and Liu, X.: "Robust stability of two-dimensional uncertain discrete systems," IEEE Signal Processing Letters, May 2003, 10, pp. $133-136$ 